\title{
Knockdown of SCARA5 inhibits PDGF-BB-induced vascular smooth muscle cell proliferation and migration through suppression of the PDGF signaling pathway
}

\author{
JIANGTAO ZHAO ${ }^{1}$, LIGUO JIAN ${ }^{1}$, LIHUA ZHANG ${ }^{1}$, TONGBIN DING ${ }^{1}$, XIAOWEI LI $^{1}$, DONG CHENG ${ }^{1}$, \\ SHAOHUI NIU ${ }^{1}$, LIQIANG SUN ${ }^{1}$, EN LI ${ }^{1}$, SHICHAO LIU ${ }^{1}$, YOUXU JIANG ${ }^{1}$ and LU LIU ${ }^{2}$ \\ ${ }^{1}$ The Second Ward of Cardiovascular Department, The Second Affiliated Hospital of Zhengzhou University; \\ ${ }^{2}$ Department of Nephrology, The First Affiliated Hospital of Zhengzhou University, Zhengzhou, Henan 450000, P.R. China
}

Received May 5, 2015; Accepted March 4, 2016

DOI: $10.3892 / \mathrm{mmr} .2016 .5074$

\begin{abstract}
Vascular smooth muscle cell (VSMC) proliferation and migration are critical in the progression of atherosclerosis and can be induced by platelet-derived growth factor (PDGF). Several studies have demonstrated that scavenger receptor class A, member 5 (SCARA5) is important in cancer cell migration and invasion. However, the role of SCARA5 in VSMCs remains to be elucidated in the development of atherosclerosis. Therefore, the role of SCARA5 was investigated in PDGF-BB-stimulated VSMC proliferation and migration. In the present study, it was shown that SCARA5 expression was enhanced by PDGF-BB in human aortic smooth muscle cells (HASMCs). Knockdown of SCARA5 by small interfering (si)RNA significantly inhibited PDGF-BB-induced HASMC proliferation and migration. Furthermore, siRNA-SCARA5 significantly inhibited the phosphorylation of PDGF receptor (PDGFR) $\beta$, AKT and extracellular signal-regulated kinase 1/2 in PDGF-BB-stimulated HASMCs. In conclusion, this study demonstrated that knockdown of SCARA5 inhibits PDGF-BB-induced HASMC proliferation and migration through suppression of the PDGF signaling pathway. Thus, SCARA5 may be a novel therapeutic target for preventing or treating vascular diseases involving VSMC proliferation and migration.
\end{abstract}

Correspondence to: Dr Jiangtao Zhao, The Second Ward of Cardiovascular Department, The Second Affiliated Hospital of Zhengzhou University, 2 Eight Road, Jinshui, Zhengzhou, Henan 450000, P.R. China

E-mail: jiangtao_zhaoz@163.com

Key words: scavenger receptor class A, member 5, platelet-derived growth factor-BB, human aortic smooth muscle cells, proliferation, migration

\section{Introduction}

Atherosclerosis is a chronic inflammatory response to injury in the arterial wall (1). It is harmful to human health and responsible for the majority of fatalities in the senior population (2). The initial step of atherogenesis is intimal injury and subsequent platelet aggregation and leukocyte invasion beneath the endothelial monolayer. Furthermore, through macrophage activation and foam cell formation, vascular smooth muscle cell (VSMC) proliferation and migration lead to atherosclerosis (3).

Increased proliferation and migration of VSMCs are critical events in the pathophysiology of atherosclerosis (4). The proliferation of VSMCs can be induced by cytokines and growth factors, such as tumor necrosis factor- $\alpha$, platelet-derived growth factor (PDGF) and transforming growth factor (TGF) $-\beta$ (3). It has been reported that PDGF initiates numerous biological effects via the activation of intracellular signal transduction pathways that are critical in the proliferation and migration of VSMCs (5). Therefore, preventing PDGF-mediated VSMC proliferation and migration may be an important therapeutic approach for atherosclerosis.

Class A scavenger receptors (SR-As) are cell surface receptors that bind a range of ligands, including modified low-density lipoproteins and nucleic acids (6). The SR-A types found in mammals are macrophage scavenger receptor type A (SR-A, SCARA1), MARCO (SCARA2), CSR1 (SCARA3), SRCL4 (SCARA4) and SCARA5 (7). Several studies have demonstrated that SCARA5 is critical in cancer cell migration and invasion (8-10). More recently, one study showed that catechin supplementation reduced the atherosclerotic lesion area, and downregulated the expression levels of SCARA5 (11). These results suggest that SCARA5 may be involved in atherosclerosis. However, the role of SCARA5 in VSMCs remains to be elucidated in the development of atherosclerosis. Therefore, the role of SCARA5 in PDGF-BB-stimulated HASMC proliferation and migration was investigated.

\section{Materials and methods}

Cell culture. Human aortic smooth muscle cells (HASMCs) were obtained from the American Type Culture Collection 
(Manassas, VA, USA). Cells were cultured in SmGM-2 growth media (Lonza, Basel, Switzerland) in 5\% fetal bovine serum (Gibco; Thermo Fisher Scientific, Inc., Waltham, MA, USA). at $37^{\circ} \mathrm{C}$ in a humidified $5 \% \mathrm{CO}_{2}$ incubator. Cells from passages 3 to 5 were used in the experiments.

Reverse transcription-quantitative polymerase chain reaction $(R T-q P C R)$ analysis. Total RNA was extracted from HASMCs using the RNA plus kit (Takara Biotechnologies Inc., Dalian, China). cDNA was synthesized from $0.5 \mu \mathrm{g}$ of total RNA with superscriptor reverse transcriptase (Invitrogen; Thermo Fisher Scientific, Inc.). The levels of gene mRNA transcripts were analyzed using the specific primers and SYBR Green I reagent (Takara Biotechnologies Inc.) and the RT-PCR kit (Takara Biotechnologies Inc.), on the Bio-Rad iQ5 Quantitative PCR system (Bio-Rad, Hercules, CA, USA), according to the manufacturer's instructions. The following primers were used: SCARA5, 5'-CAGCTGGTTTCTTACCACGTAT-3' (sense), 5'-GCACAAGTTCTCCCACACTTAG-3' (antisense); and $\beta$-actin $5^{\prime}$-CCGTGAAAAGATGACCCAGATC-3' (sense), 5'-CACAGCCTGGATGGCTACGT-3' (antisense) (all obtained from Takara Biotechnologies Inc.). For relative quantification, the levels of individual gene mRNA transcripts were firstly normalized to the control $\beta$-actin. Subsequently, the differential expression of these genes was analyzed by the DDCt method and expressed as the fold changes. The PCR procedure was as follows: Initial denaturation at $94^{\circ} \mathrm{C}$ for $5 \mathrm{~min} ; 40$ cycles of denaturation at $94^{\circ} \mathrm{C}$ for $30 \mathrm{sec}$, annealing at $55^{\circ} \mathrm{C}$ for $30 \mathrm{sec}$ and extension at $72^{\circ} \mathrm{C}$ for $20 \mathrm{sec}$; and the melt curve was analyzed from 65 to $95^{\circ} \mathrm{C}$. Expression levels of the relative genes were calculated using the $2^{-\Delta \Delta \mathrm{Cq}}$ method (12) and with $\beta$-actin mRNA as an internal control.

Western blotting. Total protein extracts were prepared using radioimmunoprecipitation assay (RIPA) lysis buffer (Beyotime Institute of Biotechnology, Nantong, China) according to the manufacturer's instructions. Protein concentration was determined by a bicinchoninic acid protein assay (Sangon Biotech, Shanghai, China) using bovine serum albumin (Gibco; Thermo Fisher Scientific, Inc.) as the standard. Aliquots $(30 \mu \mathrm{l})$ were separated on a $10 \%$ sodium dodecyl sulfate-polyacrylamide gel electrophoresis and transferred onto polyvinylidene difluoride membranes (Whatman Schleicher \& Schuell, Middlesex, UK). Membranes were blocked with Tris-buffered saline (TBS) containing $1 \%(\mathrm{w} / \mathrm{v})$ non-fat dry milk and $0.1 \%(\mathrm{v} / \mathrm{v})$ Tween-20 (TBST) for $>2 \mathrm{~h}$. After blocking, the target proteins were probed with the following primary antibodies overnight at $4^{\circ} \mathrm{C}$ : Rabbit anti-SCARA5 $(1: 1,500$; sc-98123), mouse anti-platelet-derived growth factor receptor $\beta$ (PDGFR $\beta$ ) $(1: 1,500 ;$ sc-19995), mouse anti-AKT $(1: 2,000 ;$ sc-5298), rabbit anti-phospho-AKT (1:2,000; sc-135650), rabbit anti-extracellular signal-regulated kinase (ERK) $(1: 2,000$; sc-292838), mouse anti-phospho-ERK (1:2,000; sc-81492) and anti- $\beta$-actin (1:1500; sc-47778) (all from Santa Cruz Biotechnology, Inc., Santa Cruz, CA, USA). Subsequently, the blots were washed with TBST and incubated with Subsequently, the blots were washed with TBST and incubated with horseradish peroxidase (HRP)-conjugated goat anti-mouse immunoglobulin G (sc-395761) and bovine
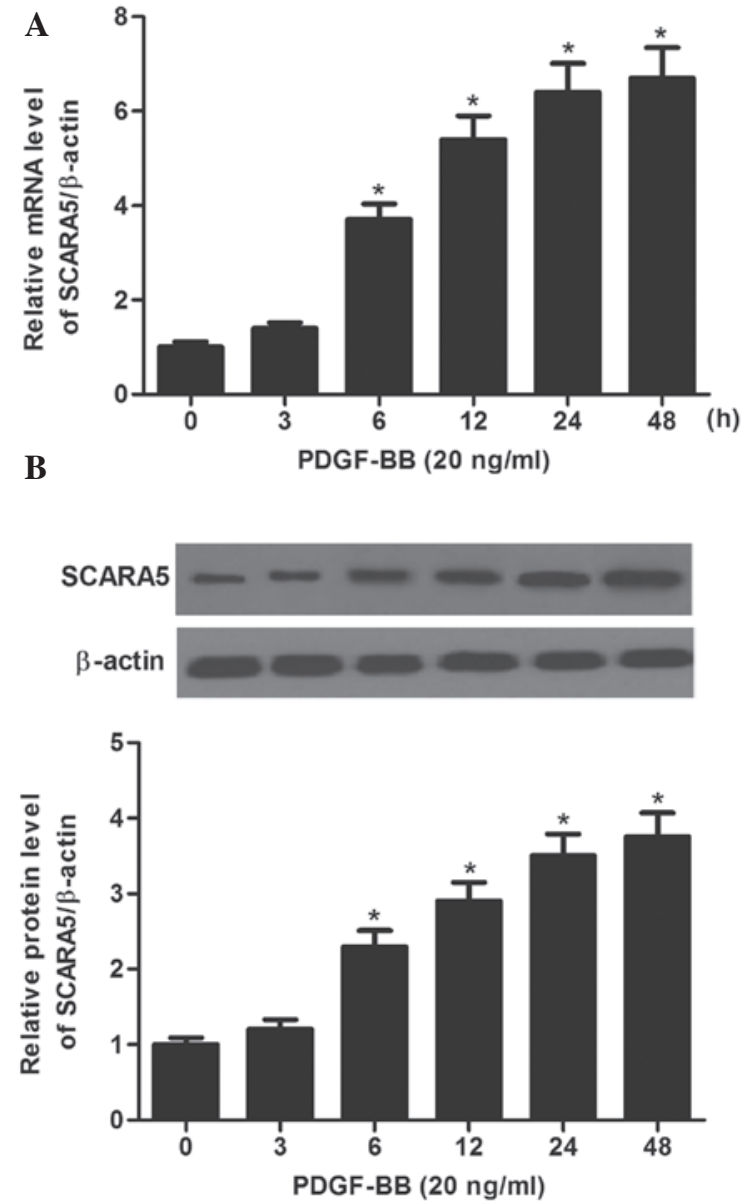

Figure 1. PDGF-BB induced SCARA5 expression in HASMCs. (A) Time-dependent induction of SCARA5 mRNA expression by PDGF-BB. (B) Time-dependent induction of SCARA5 protein expression by PDGF-BB. Results are presented as the mean \pm standard deviation from three independent experiments performed in duplicate. Relative expression is expressed in arbitrary units. ${ }^{*} \mathrm{P}<0.05$ compared with no PDGF-BB treatment. PDGF-BB, platelet-derived growth factor BB; SCARA5, scavenger receptor class A, member 5; HASMCs, human aortic smooth muscle cells.

anti-rabbit horseradish peroxidase conjugated secondary antibody (sc-2370) (both 1:3,000; Santa Cruz Biotechnology, Inc.) for $1 \mathrm{~h}$. After washing, the sites of antibody binding were visualized by chemiluminescence (Boehringer Mannheim, Mannheim, Germany). $\beta$-actin protein levels were used as an endogenous control to allow the normalization of target proteins. The relative protein expression levels were quantified using Image-Pro Plus 6.0 software (Media Cybernetics, Silver Spring, MD, USA) and normalized to $\beta$-actin.

SCARA5 small interfering (si)RNA transfection. SCARA5 siRNA was designed by Genepharma (Shanghai, China). Sequences corresponding to the siRNA of SCARA5 were: Sense, 5'-GCUCCAUCUGUGAGGAUUCdTdT-3' and antisense, 5'-GAAUCCUCAGAUGGAGdTdT-3'. For in vitro transfection, HASMCs were seeded at a density of $1 \times 10^{4}$ cells/well in each well of a 96-well microplate and grown for $24 \mathrm{~h}$ to reach $60 \%$ confluence. The cells were transfected with either siRNA-scramble or siRNA-SCARA5 using HiPerFect Transfection reagent (Qiagen) according to the manufacturer's instructions. A scramble Stealth RNAi duplex served as a negative control (sense, 5'-UUCUCGAAC 
A
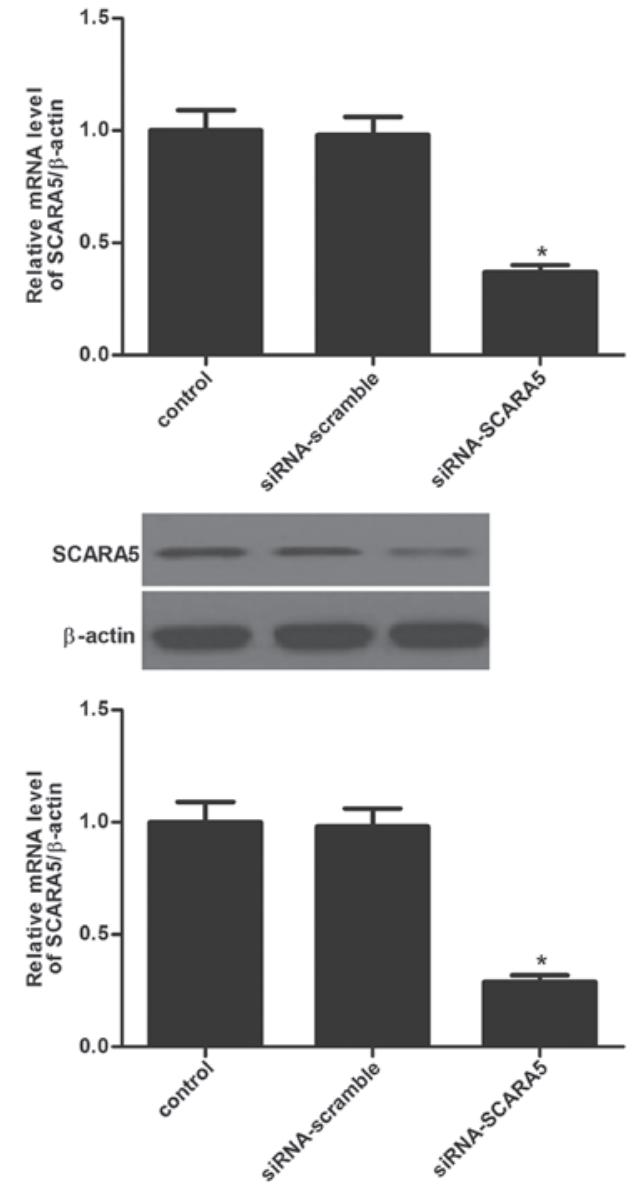

Figure 2. Expression of SCARA5 was significantly decreased in siRNA-SCRAR5-transfected HASMCs. HASMCs were transfected with siRNA-scramble and siRNA-SCRAR5, respectively. The corresponding transfection efficiency was detected by reverse transcription-quantitative polymerase chain reaction and western blot analysis. (A) SCARA5 mRNA expression in HASMCs; (B) SCARA5 protein expression in HASMCs; Results are presented as the mean \pm standard deviation from three independent experiments performed in duplicate. ${ }^{*} \mathrm{P}<0.05$ vs. the untreated cells. PDGF-BB, platelet-derived growth factor BB; SCARA5, scavenger receptor class A, member 5; HASMCs, human aortic smooth muscle cells. siRNA, small interfering RNA.

\section{GUGUCACGUdTdT-3'; and antisense, 5'-ACGUACACGUUC GGAGAAdTdT-3').}

HASMC proliferation assay. HASMC proliferation was measured by an MTT assay. In brief, HASMCs $\left(4 \times 10^{4} \mathrm{cells} / \mathrm{ml}\right)$ were growth-arrested in a 96-well microplate. After being grown to $60 \%$ confluence, HASMCs were incubated in serum-free medium for $24 \mathrm{~h}$ prior to stimulation with $20 \mathrm{ng} / \mathrm{ml}$ PDGF-BB and/or siRNA-SCARA5 in serum free medium. After $24 \mathrm{~h}, 0.5 \mu \mathrm{g} / \mathrm{ml}$ MTT Sigma-Aldrich (St. Louis, MO, USA) was added and incubated for $4 \mathrm{~h}$. Subsequently, $400 \mu \mathrm{l}$ dimethyl sulfoxide was added to dissolve the formazan crystals formed. The absorbance value was measured at $450 \mathrm{~nm}$ using a microplate reader (model 680, Bio-Rad).

HASMC migration assay. HASMCs migration was measured with the Transwell migration assay. In brief, HASMCs were resuspended in serum-free Dulbecco's modified Eagle's medium (Gibco; Thermo Fisher Scientific, Inc.), and placed in the upper compartment of Transwell chambers, while the

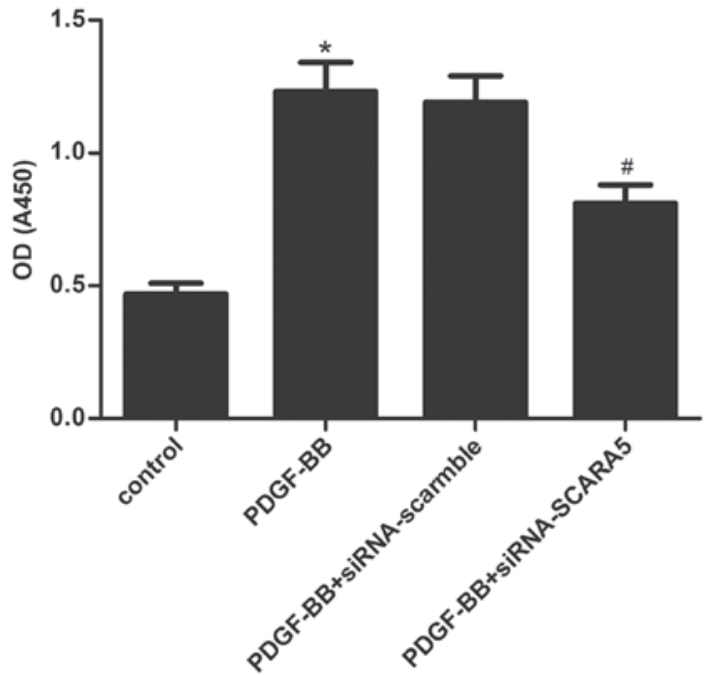

Figure 3. Effects of SCARA5 on PDGF-BB-induced proliferation of HASMCs. Cell proliferation was assessed by an MTT assay. The cells were incubated in serum-free medium for $24 \mathrm{~h}$ prior to stimulation with $20 \mathrm{ng} / \mathrm{ml}$ PDGF-BB and/or siRNA-SCARA5 in serum free medium. siRNA-SCRAR5 inhibited PDGF-BB-induced HASMC proliferation. ${ }^{*} \mathrm{P}<0.05$ vs. the untreated cells. ${ }^{~} \mathrm{P}<0.05$ vs. PDGF-BB treatment. PDGF-BB, platelet-derived growth factor BB; SCARA5, scavenger receptor class A, member 5; HASMCs, human aortic smooth muscle cells. siRNA, small interfering RNA; OD, optical density.

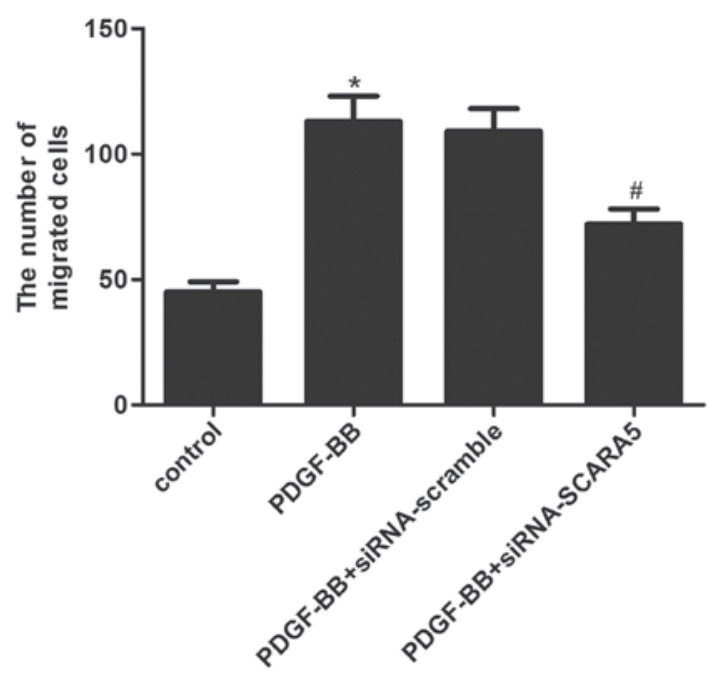

Figure 4. Effects of SCARA5 on PDGF-BB-induced migration of HASMCs. Cell migration was assessed by the Transwell assay. The cells were incubated in serum-free medium for $24 \mathrm{~h}$ prior to stimulation with $20 \mathrm{ng} / \mathrm{ml}$ PDGF PDGF and/or siRNA-SCARA5 in serum free medium. siRNA-SCRAR5 inhibited PDGF-BB-induced HASMC migration. ${ }^{*} \mathrm{P}<0.05$ vs. untreated cells ${ }^{\#} \mathrm{P}<0.05$ vs. PDGF-BB treatment. PDGF-BB, platelet-derived growth factor BB; SCARA5, scavenger receptor class A, member 5; HASMCs, human aortic smooth muscle cells. siRNA, small interfering RNA.

lower chamber was supplemented with serum-free medium (Sigma-Aldrich) containing PDGF-BB (Sigma-Aldrich) as a positive control. After incubation for $16 \mathrm{~h}$ at $37^{\circ} \mathrm{C}$, the Transwells (Sigma-Aldrich) were washed twice with phosphate-buffered saline. The cells from the top side of the filter were removed with a cotton swab, and cells on the bottom side of the filter were stained for $30 \mathrm{~min}$ with Gill hematoxylin (Sigma-Aldrich). The number of cells on the lower 


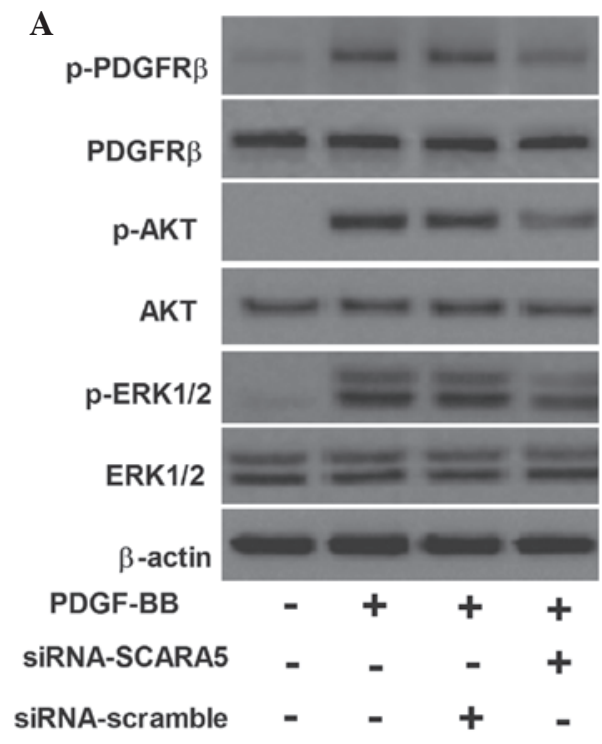

$\mathbf{B}$

C

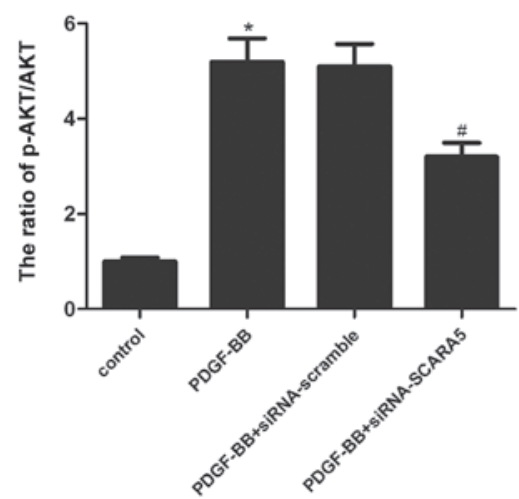

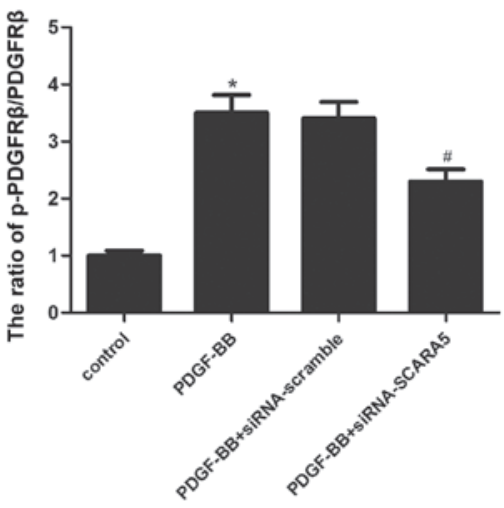

D

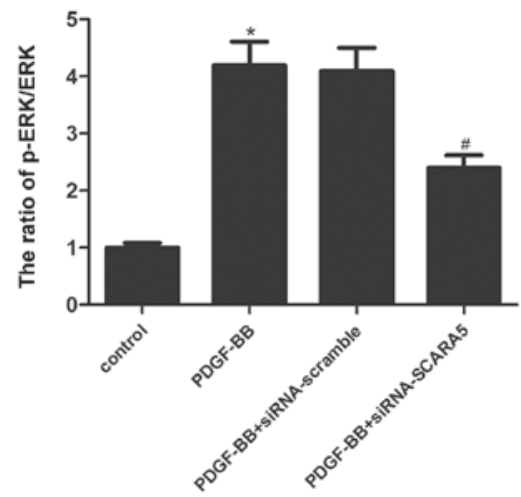

Figure 5. Effects of SCARA5 on PDGF-BB-induced PDGFR $\beta$, AKT, and ERK1/2 phosphorylation. (A) The cells were incubated in serum-free medium for $24 \mathrm{~h}$ prior to stimulation with $20 \mathrm{ng} / \mathrm{ml}$ PDGF-BB and/or siRNA-SCARA5 in serum free medium at $37^{\circ} \mathrm{C}$ for 5 min (for PDGFR $\beta$, AKT and ERK1/2 phosphorylation). The cells were lysed, and proteins were analyzed using 7.5 and $10 \%$ sodium dodecyl sulfate-polyacrylamide gel electrophoresis and immunoblotting. The relative protein expression levels of (B) p-PDGFR $\beta$, (C) p-AKT and (D) p-ERK1/2 were quantified using Image-Pro Plus 6.0 software and normalized to $\beta$-actin. Representative data from three different experiments are presented. ${ }^{*} \mathrm{P}<0.05$ vs. control. ${ }^{"} \mathrm{P}<0.05$ vs. PDGF-BB treatment. SCARA5, scavenger receptor class $\mathrm{A}$, member 5; PDGF-BB, platelet-derived growth factor BB; PDGFR $\beta$, platelet-derived growth factor receptor $\beta$; ERK1/2, extracellular signal-regulated kinase 1/2; siRNA, small interfering RNA.

surface in five random fields was counted under a microscope (SZX7-1063, Olympus, Tokyo, Japan). in order to determine the average number of cells that had migrated.

Statistical analysis. Values are presented as the mean \pm standard deviation. Data were analyzed using one-way analysis of variance followed by Fisher's Least Significant Differences post hoc test. All analyses were conducted using 13.0 SPSS software (SPSS Inc., Chicago, IL, USA). P $<0.05$ was considered to indicate a statistically significant difference.

\section{Results}

SCARA5 is enhanced by growth factor PDGF-BB. It has been reported that PDGF-BB promotes the dedifferentiation and proliferation of SMCs, and is upregulated at the site of vascular injury and in atherosclerotic plaque specimens (13). Thus, the present study examined the response of SCARA5 to PDGF-BB in cultured HASMCs. As shown in Fig. 1A, the
mRNA level of SCARA5 was increased in a time-dependent manner in response to $20 \mathrm{ng} / \mathrm{ml}$ PDGF-BB. Similarly, western blotting demonstrated that the level of SCARA5 protein was also increased by PDGF-BB (Fig. 1B).

Knockdown of SCARA5 markedly inhibits HASMC proliferation in vitro. Evidence shows that HASMCs are critical in the development of atherosclerosis and that SCARA5 is notably reduced in PDGF-stimulated HASMCs. Thus, siRNA-SCARA5 was designed and transfected into the HASMCs in order to investigate the role of SCARA5 in HASMC. The transfection efficiency was determined by RT-qPCR and western blot analysis in HASMCs. As shown in Fig. 2A and B, the mRNA and protein expression levels of SCARA5 were significantly decreased in HASMCs. Moreover, an MTT assay was performed to identify the effect of SCARA5 on HASMC proliferation. HASMCs were pretreated with siRNA-SCARA5 or siRNA-scramble for $24 \mathrm{~h}$ and then stimulated with PDGF-BB $(20 \mathrm{ng} / \mathrm{ml})$ for $24 \mathrm{~h}$. 
As indicated in Fig. 3, PDGF-BB treatment significantly increased the proliferation of HASMCs, compared with the untreated cells; however, siRNA-SCARA5 significantly prevented PDGF-BB-induced HASMC proliferation. These results suggested that SCARA5 facilitates HASMC proliferation in vitro.

Knockdown of SCARA5 markedly retards HASMC migration in vitro. A Transwell migration assay was conducted to investigate the effect of SCARA5 on HASMC migration. As shown in Fig. 4, the Transwell assay revealed that treatment with PDGF-BB markedly increased the number of HASMCs that migrated through the Transwell chamber, while, siRNA-SCARA5 significantly reduced the number of cells that migrated following PDGF-BB stimulation. These results suggested that SCARA5 facilitates HASMC migration in vitro.

Knockdown of SCARA5 inhibits HASMC proliferation and migration through the suppression of the PDGF signaling pathway. To investigate the mechanisms of SCARA5-promoted HASMC proliferation and migration, it was examined whether SCARA5 affected the phosphorylation of AKT and ERK1/2 in PDGF-BB-stimulated HASMCs. As shown in Fig. 5, treatment with PDGF-BB markedly increased the phosphorylation of PDGFR $\beta$ in HASMCs. In addition, the phosphorylation of AKT and ERK1/2 were also increased by PDGF-BB in HASMCs. While, siRNA-SCARA5 significantly inhibited AKT and ERK1/2 phosphorylation by PDGF-BB in HASMCs, which was associated with a reduction in PDGFR $\beta$ phosphorylation by PDGF-BB stimulation.

\section{Discussion}

VSMC proliferation and migration are critical in the progression of atherosclerotic lesions. Inhibition of VSMC proliferation and migration is an important therapeutic strategy for atherosclerosis (14). In the present study, it was demonstrated that SCARA5 expression was enhanced by PDGF-BB in HASMCs. Knockdown of SCARA5 by siRNA significantly inhibited PDGF-BB-induced HASMC proliferation and migration. Furthermore, siRNA-SCARA5 significantly inhibited AKT and ERK1/2 phosphorylation by PDGF-BB in HASMCs.

SR-As are membrane glycoproteins that can form homotrimers. This receptor was originally defined by its ability to mediate the accumulation of lipids in macrophages. Recently, Syväranta et al (15) showed that the mRNA expression level of SR-A1 was upregulated in stenotic human aortic valves. In this study, it was demonstrated that SCARA5 expression was enhanced by PDGF-BB in HASMCs. These results suggest that SCARA5 may be important in the development of atherosclerosis.

PDGF and its $\beta$ receptor subtype (PDGFR $\beta)$, which is abundantly expressed in VSMCs, are significantly upregulated and activated at sites of vascular injury $(16,17)$. In this study, PDGF was used as a proliferative agent as studies have demonstrated that PDGF is a critical mediator of VSMC growth $(18,19)$. In line with these previous studies, it was demonstrated that PDGF-BB increased HASMC proliferation and migration. However, siRNA-SCARA5 inhibited the proliferation and migration of PDGF-BB-stimulated HASMCs.

PDGF-BB, one of the most potent mitogens and chemoattractants for VSMCs, is important in the development of atherosclerosis (16). Upon binding to PDGFR $\beta$ on VSMCs, PDGF-BB initiates a multitude of biological effects through the activation of ERK1/2 MAP kinase. ERK1/2 transduces mitogenic signals to the nucleus by phosphorylating and activating specific transcription factors, thereby leading to the formation of hyperplastic lesions in response to vascular injury $(20,21)$. ERK1/2 has been involved in PDGF-induced upregulation of the neuron-derived orphan receptor-1 in VSMCs that is required for cell cycle progression (22). Moreover, PDGF-BB binds to PDGFR $\beta$ and activates the phosphatidylinositol 3-kinase (PI3K)/AKT pathway in VSMCs. Activated AKT has been implicated in the PDGF-BB-induced proliferation, migration and changes in the cytoskeleton of VSMCs $(23,24)$. A PDGFR $\beta$ antagonist or protein kinase inhibitor suppressed VMSC proliferation in vitro and prevented cardiovascular disorders in several animal experiments $(25,26)$. In this study, it was demonstrated that PDGF-BB markedly increased the phosphorylation of PDGFR $\beta$, AKT and ERK $1 / 2$ in PDGF-BB-stimulated HASMCs. While, siRNA-SCARA5 inhibited PDGF-BB-induced phosphorylation of PDGFR $\beta$, AKT and ERK1/2. These results indicate that inhibiting PDGF-BB-induced activation of the PDGF signaling pathway may have contributed to the inhibition of HASMC proliferation and migration exerted by siRNA-SCARA5.

In conclusion, this study demonstrated that knockdown of SCARA5 significantly inhibited HASMC proliferation and migration induced by PDGF-BB. In addition, knockdown of SCARA5 notably inhibited the activation of the PDGF signaling pathway. Therefore, SCARA5 may be a novel therapeutic target for preventing or treating vascular diseases involving VSMC proliferation and migration.

\section{References}

1. Ross R and Agius L: The process of atherogenesis-cellular and molecular interaction: From experimental animal models to humans. Diabetologia 35 (Suppl 2): S34-S40, 1992.

2. Wang JC and Bennett M: Aging and atherosclerosis: Mechanisms, functional consequences and potential therapeutics for cellular senescence. Circ Res 111: 245-259, 2012.

3. Ross R: Atherosclerosis-an inflammatory disease. New Engl J Med 340: 115-126, 1999.

4. Owens GK: Regulation of differentiation of vascular smooth muscle cells. Physiol Rev 75: 487-517, 1995.

5. Heldin $\mathrm{CH}$ and Westermark B: Mechanism of action and in vivo role of platelet-derived growth factor. Physiol Rev 79: 1283-1316, 1999.

6. Peiser L and Gordon S: The function of scavenger receptors expressed by macrophages and their role in the regulation of inflammation. Microbes Infect 3: 149-159, 2001.

7. Whelan FJ, Meehan CJ, Golding GB, McConkey BJ and Bowdish DM: The evolution of the class A scavenger receptors. BMC Evol Biol 12: 227-237, 2012.

8. Liu J, Hu G, Chen D, Gong AY, Soori GS, Dobleman TJ and Chen XM: Suppression of SCARA5 by Snaill is essential for EMT-associated cell migration of A549 cells. Oncogenesis 2: e73, 2013.

9. Yan N, Zhang S, Yang Y, Cheng L, Li C, Dai L, Dai L, Zhang X, Fan P, Tian H, et al: Therapeutic upregulation of Class A scavenger receptor member 5 inhibits tumor growth and metastasis. Cancer Sci 103: 1631-1639, 2012. 
10. Huang J, Zheng DL, Qin FS, Cheng N, Chen H, Wan BB, Wang YP, Xiao HS and Han ZG: Genetic and epigenetic silencing of SCARA5 may contribute to human hepatocellular carcinoma by activating FAK signaling. J Clin Invest 120 : 223-241, 2010.

11. Auclair S, Milenkovic D, Besson C, Chauvet S, Gueux E, Morand C, Mazur A and Scalbert A: Catechin reduces atherosclerotic lesion development in apo E-deficient mice: A transcriptomic study. Atherosclerosis 204: e21-e27, 2009.

12. Chadjichristos CE, Morel S, Derouette JP, Sutter E, Roth I, Brisset AC, Bochaton-Piallat ML and Kwak BR: Targeting connexin 43 prevents platelet-derived growth factor-BB-induced phenotypic change in porcine coronary artery smooth muscle cells. Circ Res 102: 653-660, 2008.

13. Livak KJ and Schmittgen TD: Analysis of relative gene expression data using real-time quantitative PCR and the 2(-Delta Delta C(T)) Method. Methods 25: 402-408, 2001.

14. Andrés V: Control of vascular cell proliferation and migration by cyclin-dependent kinase signalling: New perspectives and therapeutic potential. Cardiovasc Res 63: 11-21, 2004.

15. Syväranta S, Alanne-Kinnunen M, Oörni K, Oksjoki R, Kupari M, Kovanen PT and Helske-Suihko S: Potential pathological roles for oxidized low-density lipoprotein and scavenger receptors SR-AI, CD36 and LOX-1 in aortic valve stenosis. Atherosclerosis 235: 398-407, 2014

16. Raines EW: PDGF and cardiovascular disease. Cytokine Growth Factor Rev 15: 237-254, 2004.

17. Abe J, Deguchi J, Takuwa Y, Hara K, Ikari Y, Tamura T, Ohno M and Kurokawa K: Tyrosine phosphorylation of platelet derived growth factor beta receptors in coronary artery lesions: Implications for vascular remodelling after directional coronary atherectomy and unstable angina pectoris. Heart 79: 400-406, 1998.

18. Jawien A, Bowen-Pope DF, Lindner V, Schwartz SM and Clowes AW: Platelet-derived growth factor promotes smooth muscle migration and intimal thickening in a rat model of balloon angioplasty. J Clin Invest 89: 507-511, 1992.
19. Bilder G, Wentz T, Leadley R, Amin D, Byan L, O'Conner B, Needle S, Galczenski H, Bostwick J, Kasiewski C, et al: Restenosis following angioplasty in the swine coronary artery is inhibited by an orally active PDGF-receptor tyrosine kinase inhibitor, RPR101511A. Circulation 99: 3292-3299, 1999.

20. Lannoy M, Slove S, Louedec L, Choqueux C, Journé C, Michel JB and Jacob MP: Inhibition of ERK1/2 phosphorylation: A new strategy to stimulate elastogenesis in the aorta. Hypertension 64: 423-430, 2014

21. Mii S, Khalil RA, Morgan K, Ware JA and Kent KC: Mitogen-activated protein kinase and proliferation of human vascular smooth muscle cells. Am J Physiol 270: H142-H150, 1996.

22. Nomiyama T, Nakamachi T, Gizard F, Heywood EB, Jones KL, Ohkura N, Kawamori R, Conneely OM and Bruemmer D: The NR4A orphan nuclear receptor NOR1 is induced by platelet-derived growth factor and mediates vascular smooth muscle cell proliferation. J Biol Chem 281: 33467-33476, 2006.

23. Goncharova EA, Ammit AJ, Irani C, Carroll RG, Eszterhas AJ, Panettieri RA and Krymskaya VP: PI3K is required for proliferation and migration of human pulmonary vascular smooth muscle cells. Am J Physiol Lung Cell Mol Physiol 283: L354-L363, 2002.

24. Choi KH, Kim JE, Song NR, Son JE, Hwang MK, Byun S, Kim JH, Lee KW and Lee HJ: Phosphoinositide 3-kinase is a novel target of piceatannol for inhibiting PDGF-BB-induced proliferation and migration in human aortic smooth muscle cells. Cardiovasc Res 85: 836-844, 2010.

25. Lipson KE, Pang L, Huber LJ, Chen H, Tsai JM, Hirth P, Gazit A, Levitzki A and McMahon G: Inhibition of platelet-derived growth factor and epidermal growth factor receptor signaling events after treatment of cells with specific synthetic inhibitors of tyrosine kinase phosphorylation. J Pharmacol Exp Ther 285: 844-852, 1998.

26. Myllärniemi M, Frösen J, Ramirez LG, Buchdunger E, Lemström K and Häyry P: Selective tyrosine kinase inhibitor for the platelet-derived growth factor receptor in vitro inhibits smooth muscle cell proliferation after reinjury of arterial intima in vivo. Cardiovasc Drug Ther 13: 159-168, 1999. 